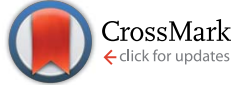

Cite this: J. Mater. Chem. A, 2015, 3, 7974

Received 12th February 2015

Accepted 3rd March 2015

DOI: $10.1039 / c 5 t a 01183 c$

www.rsc.org/MaterialsA

\section{Tailoring the dispersion of multiwall carbon nanotubes in co-continuous PVDF/ABS blends to design materials with enhanced electromagnetic interference shielding}

\begin{abstract}
Goutam Prasanna Kar, $\uparrow$ Sourav Biswas, $\uparrow$ Rani Rohini and Suryasarathi Bose*
Highly conducting composites were derived by selectively localizing multiwall carbon nanotubes (MWNTs) in co-continuous PVDF/ABS (50/50, wt/wt) blends. The electrical percolation threshold was obtained between 0.5 and $1 \mathrm{wt} \%$ MWNTs as manifested by a dramatic increase in the electrical conductivity by about six orders of magnitude with respect to the neat blends. In order to further enhance the electrical conductivity of the blends, the MWNTs were modified with amine terminated ionic liquid (IL), which, besides enhancing the interfacial interaction with PVDF, facilitated the formation of a network like structure of MWNTs. This high electrical conductivity of the blends, at a relatively low fraction (1 wt\%), was further explored to design materials that can attenuate electromagnetic (EM) radiation. More specifically, to attenuate the EM radiation by absorption, a ferroelectric phase was introduced. To accomplish this, barium titanate (BT) nanoparticles chemically stitched onto graphene oxide (GO) sheets were synthesized and mixed along with MWNTs in the blends. Intriguingly, the total EM shielding effectiveness (SE) was enhanced by ca. $10 \mathrm{~dB}$ with respect to the blends with only MWNTs. In addition, the effect of introducing a ferromagnetic phase $\left(\mathrm{Fe}_{3} \mathrm{O}_{4}\right)$ along with IL modified MWNTs was also investigated. This study opens new avenues in designing materials that can attenuate $\mathrm{EM}$ radiation by selecting either a ferroelectric $(\mathrm{BT}-\mathrm{GO})$ or a ferromagnetic phase $\left(\mathrm{Fe}_{3} \mathrm{O}_{4}\right)$ along with intrinsically conducting nanoparticles (MWNTs).
\end{abstract}

\section{Introduction}

Blending polymers with different physical properties opens new avenues in designing materials with a unique set of properties. In general, due to the low entropy of mixing, ${ }^{1}$ most polymer pairs are thermodynamically immiscible. It is generally agreed that specific interactions between the blend components govern their miscibility ${ }^{2-5}$ and ultimately dictate the properties of the blends. Besides the properties of the component polymers, the overall performance of the blends is largely governed by the morphology thus formed by blending. ${ }^{6,7}$ Most often, matrix dispersed and co-continuous morphologies are observed by mixing two polymers in different ratios. ${ }^{8}$ Increasing attention has been paid to co-continuous structures, which offer a unique combination of properties offered by each of the components. ${ }^{9}$

An alternate route to develop materials with tailored properties is by incorporating nanoparticles into the polymer matrix. Polymer nanocomposites have drawn considerable research interest over the past decade in view of their significant

Department of Materials Engineering, Indian Institute of Science, Bangalore-560012, India. E-mail: sbose@materials.iisc.ernet.in; Tel: +91-80-2293 3407

$\dagger$ GPK and SB made equal contribution to this work. improvement in physical, thermal, mechanical and electronic properties of the matrix polymer. In recent years, more focus was laid on designing flexible and lightweight polymer based conducting composites for many electronic applications. Among different nanoparticles, carbon nanotubes (CNTs) attracted a great deal of interest owing to their extraordinary combination of properties. ${ }^{\mathbf{1 0}}$ The high aspect ratio of CNTs results in a low percolation threshold in polymeric nanocomposites, which makes them ideal candidates for designing and developing conducting composites. ${ }^{\mathbf{9}, 11,12}$ However, the key challenge is their efficient dispersion in the matrix polymer and a great deal of research is focused on developing various strategies to render effective dispersion and also to facilitate network-like structures at relatively low concentration in the composites. ${ }^{10}$ The CNTs tend to agglomerate due to inter-tube van der Waals' interactions leading to a reduced 'effective aspect ratio'. It is well evident that the electrical conductivity of CNTs largely depends on the ' $\pi$-conjugation' and their high aspect ratio. In contrast to chemical functionalization of CNTs, the non-covalent approach preserves the integrity of the nanotubes and hence, the electronic properties. It is envisaged that covalent modification of CNTs affects the band gap even at the Fermi level which deteriorates the electrical properties to a 
significant extent. ${ }^{\mathbf{1 0}}$ Hence, a great deal of focus is laid on noncovalent modification of CNTs to design materials with high electrical conductivity.

Polymer blends with conducting nanoparticles have drawn considerable interest in the recent past. High electrical conductivity at a relatively low fraction of the nanoparticles in the blends can be achieved by selectively localizing the conducting nanoparticles either in a given phase or at the interface of a co-continuous blend. ${ }^{9,13-17}$ Such highly conductive materials were further exploited for many applications such as antistatic devices and EMI shielding materials ${ }^{15,16,18}$

In the recent past, the use of electronic equipment and telecommunication devices has tremendously increased. As a consequence, electromagnetic interference (EMI) and radio frequency interference (RFI) emitted by these electronic devices are quite alarming. The EM radiation emitted by the electronic devices can interfere with the precise circuitry of the nearby devices leading to adverse effects. ${ }^{19-22}$ In order to avoid serious consequences due to this interference, shielding of the EM radiation in the workspace has become vital. In order to prevent EM pollution, materials that can absorb EM radiation have become extremely important. In this context, metals are the primary choice but they suffer from high processing costs and are prone to corrosion. In this context, polymer based conducting nanocomposites have gained interest owing to their high strength to weight ratio, good processability and structural flexibility.

In recent years homopolymer based composites as EM shielding materials have been widely studied. For instance, Singh et al. showed a SE of $22 \mathrm{~dB}$ at $18 \mathrm{GHz}$ for linear density polyethylene (LDPE) based composites with $10 \mathrm{wt} \%$ MWNTs. $^{23}$ The total SE of PP nanocomposites with 7.5 vol\% MWNTs where a SE of $35.6 \mathrm{~dB}$ at $12 \mathrm{GHz}$ has been reported by Al-Saleh et al. ${ }^{24} \mathrm{In}$ the case of polyurethane (PU) based nanocomposites, Liu et al. showed a total SE of $17 \mathrm{~dB}$ for $20 \mathrm{wt} \%$ SWNTs. ${ }^{25}$ Sharma et al. showed that the EMI SE can be enhanced by using ionic liquid modified MWNTs in PVDF composites. ${ }^{26}$ More recently, the use of blends has gained interest. For instance, Rohini et al. showed a SE of $24 \mathrm{~dB}$ at $1 \mathrm{wt} \% \mathrm{NH}_{2}$ terminated MWNTs in PS/PMMA blends. ${ }^{15}$ Pawar et al. showed a SE of $17 \mathrm{~dB}$ in PC/SAN blends at $1 \mathrm{wt} \% \mathrm{NH}_{2}$ terminated MWNTs and a SE of $32.5 \mathrm{~dB}$ for the same blends with $3 \mathrm{wt} \% \mathrm{Fe}_{3} \mathrm{O}_{4}$ - $g$-MWNTs. ${ }^{18}$

In this work, various strategies were adopted to attenuate EM radiation. An immiscible pair, PVDF/ABS was selected as the model system and MWNTs were melt blended to design materials with high electrical conductivity. PVDF is an important and widely investigated polymer due to its piezoelectric properties and acrylonitrile butadiene styrene (ABS) is a tert-polymer that finds place in various applications. Recently, the application of ionic liquids (ILs) has shown promising results on structural control of the nanocomposites. ${ }^{27,28}$ ILs are organic salts with low melting points; often they are liquid at room temperature and present several intrinsic properties, like low volatility, high thermal and chemical stability, insignificant flammability, good thermal conductivity, high ionic mobility and moisture resistance. ${ }^{29,30}$ The imidazolium-based IL presents these properties together with the strong capacity of interaction with carbon based materials. The ' $\pi-\pi$ stacking' between the IL and the delocalized ' $\pi$-cloud' in MWNTs allows IL to act both as a solvent and surfactant due to the IL's amphiphilic nature. ${ }^{31}$ These features make IL ideal for exfoliating/stabilizing MWNTs, allowing broader experimental conditions, however, volatile organic solvents have the drawback of limited working temperature ranges. In order to improve the dispersion and facilitate network like structures, the MWNTs were modified with ionic liquid in this study. This is also manifested by a significant enhancement in the bulk electrical conductivity of the blends. The materials with high dielectric constant and high dielectric loss can shield EM radiation by absorption. In this regard, ferroelectric materials can be an ideal choice as losses in the ferroelectric phase are caused by electric hysteresis effects. We have synthesized a unique material where graphene oxide (GO) was chemically stitched onto barium titanate (BT) nanoparticles, a high dielectric constant material. A few compositions were prepared where this unique material (BT-GO) was incorporated along with MWNTs to attenuate EM radiation.

As mentioned earlier, high electrical conductivity, high dielectric constant and magnetic permeability are the key factors that govern the efficiency of a shielding material. Hence, a careful selection of materials can help in designing materials with enhanced EMI shielding. In light of this, we adopted a unique strategy where a three phase structure involving either a ferroelectric phase (BT-GO) or a ferromagnetic phase $\left(\mathrm{Fe}_{3} \mathrm{O}_{4}\right.$ nanoparticles) in combination with an intrinsically conducting nanoparticle (MWNTs) was used to design lightweight and flexible polymeric nanocomposites. The phase morphology, selective localization, electrical conductivity and EM shielding effectiveness were assessed here in various PVDF/ABS blends.

\section{Experimental section}

\section{Materials}

PVDF (Kynar-761, with Mw of $440000 \mathrm{~g} \mathrm{~mol}^{-1}$ ) was kindly provided by Arkema. ABS (Absolac 120, with a typical composition consisting of acrylonitrile: $24 \mathrm{wt} \%$, rubber content: 16.5 $\mathrm{wt} \%$ and styrene: $59.5 \mathrm{wt} \%$ ) was procured from Styrolution. The pristine multiwall carbon nanotubes (p-MWNTs) with an average diameter and a length of $9.5 \mathrm{~nm}$ and $1.5 \mu \mathrm{m}$, respectively, were procured from Nanocyl SA (Belgium). $\mathrm{BaTiO}_{3}$ (BT, average particle size of $100 \mathrm{~nm}$ ), $\mathrm{Fe}_{3} \mathrm{O}_{4}$ nanoparticles (with an average diameter of 50-100 nm), 1-vinylimidazole, 3-bromopropylaminehydrobromide, 3-aminopropyltriethoxysilane (APTS), $\mathrm{H}_{2} \mathrm{O}_{2}$ (30\% in water), $N, N$-dicyclohexylcarbodiimide (DCC), dry toluene, and dry $N, N$-dimethylformamide were procured from Sigma-Aldrich and were used without any further purification.

\section{Synthesis of amine terminated ionic liquid (IL)}

In a two-necked round bottomed flask, 1-vinylimidazole $(20 \mathrm{ml})$ and dry ethanol $(35 \mathrm{ml})$ were added and the mixture was stirred under a nitrogen atmosphere. 3-Bromopropylaminehydrobromide (15 g) was then added to the mixture and refluxed under stirring for $24 \mathrm{~h}$. The synthesized amine terminated ionic 
liquid was then separated by the precipitation technique with the help of ethyl acetate. Finally, ethanol was added followed by overnight vacuum drying. ${ }^{32}$

\section{Preparation of IL modified MWNTs (IL-MWNTs)}

MWNTs were modified by the as-synthesized ionic liquid as described in the above section. ${ }^{32}$ Typically, $100 \mathrm{mg}$ of MWNTs and $600 \mathrm{mg}$ of the as-synthesized ionic liquid were dispersed in $100 \mathrm{ml}$ of DMF and bath sonicated for $2 \mathrm{~h}$. The resultant mixture was then vigorously stirred at room temperature for 10 $\mathrm{h}$ and left overnight for ageing. The mixture was filtered and centrifuged to remove the ungrafted IL and finally dried under vacuum at $80{ }^{\circ} \mathrm{C}$.

\section{Synthesis of GO}

GO was synthesized by mixing graphite flakes in a mixture of concentrated $\mathrm{H}_{2} \mathrm{SO}_{4}$ and $\mathrm{H}_{3} \mathrm{PO}_{4}$ (9:1 ratio) in a water bath. $\mathrm{KMnO}_{4}$ was then added very slowly as described in our previous work. ${ }^{33}$ Then the reaction mixture was stirred in a closed reactor for $24 \mathrm{~h}$ at room temperature. After completion, the mixture was then poured into deionized (DI) water and stirred for 2 h. 35\% hydrogen peroxide $(5-10 \mathrm{ml})$ solution was added dropwise to the solution until the color turns bright yellow. The solution was then filtered. The remaining solid material was then washed in succession with DI water, $\mathrm{HCl}$ and ethanol. The solid obtained (GO) on the filter was vacuum-dried at room temperature.

\section{Synthesis of BT-GO}

BT nanoparticles were dispersed in $\mathrm{H}_{2} \mathrm{O}_{2}$ solution and bath sonicated for $15 \mathrm{~min}$. The mixture was then refluxed at $105{ }^{\circ} \mathrm{C}$ for $4 \mathrm{~h}$. The resulting hydroxylated BT nanoparticles (BT-OH) were then centrifuged and washed with water and kept for drying. The dried hydroxylated BT nanoparticles were then mixed with APTS ${ }^{34}$ followed by refluxing at $80{ }^{\circ} \mathrm{C}$ in an inert atmosphere for $24 \mathrm{~h}$. The resulting mixture was then centrifuged and washed several times with toluene and dried under vacuum. ${ }^{35}$ Thus produced amine functionalized BT nanoparticles $\left(\mathrm{BT}-\mathrm{NH}_{2}\right)$ were reacted with $\mathrm{GO}$ in dry toluene and in the presence of DCC. Free amine groups of $\mathrm{BT}-\mathrm{NH}_{2}$ react with carboxylic groups of GO forming BT-GO. The amount of GO in the hybrid was maintained as $0.1 \%$. The mixture was centrifuged, washed with toluene and dried under vacuum at $80{ }^{\circ} \mathrm{C}$.

\section{Blend preparation}

Neat blends of PVDF/ABS (50/50, wt/wt) and with different nanoparticles were prepared using a Haake minilab II under a $\mathrm{N}_{2}$ atmosphere at $230{ }^{\circ} \mathrm{C}$ temperature at $60 \mathrm{rpm}$ for 20 minutes. PVDF/ABS blends with p-MWNTs were also prepared under the same extrusion conditions mentioned above. As described above, ionic liquid modified MWNTs were prepared by a method shown in Scheme 1. The modified MWNTs were then mixed with the blends in a two step mixing protocol. In the first step, the modified MWNTs were mixed with PVDF in $N, N$ dimethylformamide followed by evaporation of the solvent. The obtained PVDF/MWNT composites were subsequently melt mixed with ABS in the second step. The total concentration of MWNTs was fixed at $1 \mathrm{wt} \%$ with respect to the total batch size. The extrusion conditions were kept the same as mentioned earlier. Similar protocols were adopted to incorporate $\mathrm{Fe}_{3} \mathrm{O}_{4}$ and BT-GO nanoparticles in the blends. Initially, these nanoparticles were mixed with PVDF in $N, N$-dimethylformamide followed by solvent evaporation. Thus obtained PVDF nanocomposites were mixed with ABS along with the ionic liquid modified MWNTs under the same extrusion conditions described above. In all the cases the samples were pre-dried at $80^{\circ} \mathrm{C}$ in a vacuum oven for $24 \mathrm{~h}$ prior to processing. Melt mixed samples were subsequently compression molded into specific shapes for different tests at $230{ }^{\circ} \mathrm{C}$ using a lab scale hydraulic press.

\section{Characterization}

Fourier transform infrared (FTIR) spectroscopy was carried out using $\mathrm{KBr}$ pellets on a Perkin-Elmer GX in the range of $4000-400 \mathrm{~cm}^{-1}$ with a resolution of $4 \mathrm{~cm}^{-1}$.

Transmission Electron Microscopy (TEM) images were acquired using a Tecnai G2F30 at $300 \mathrm{kV}$.

Morphological analysis of various blends was studied using an ULTRA 55 scanning electron microscope (SEM) with an acceleration voltage of $10 \mathrm{kV}$.

The room temperature electrical conductivity of the blends was studied using an Alpha-N Analyser, Novocontrol (Germany) in the frequency range of $0.1 \mathrm{~Hz}$ to $10 \mathrm{MHz}$. Uniformly polished compression molded disks were used as specimens and the electrical conductivity was measured across the thickness.

In order to study the electromagnetic interference SE in the $\mathrm{X}$ and Ku-band frequency range, an Anritsu MS4642A vector network analyzer (VNA) was coupled with a coax (Damaskos M07T) setup. The experimental setup was calibrated by full SOLT for both ports to avoid absorption and reflection losses due to the transmission line and sample holder. Toroidal

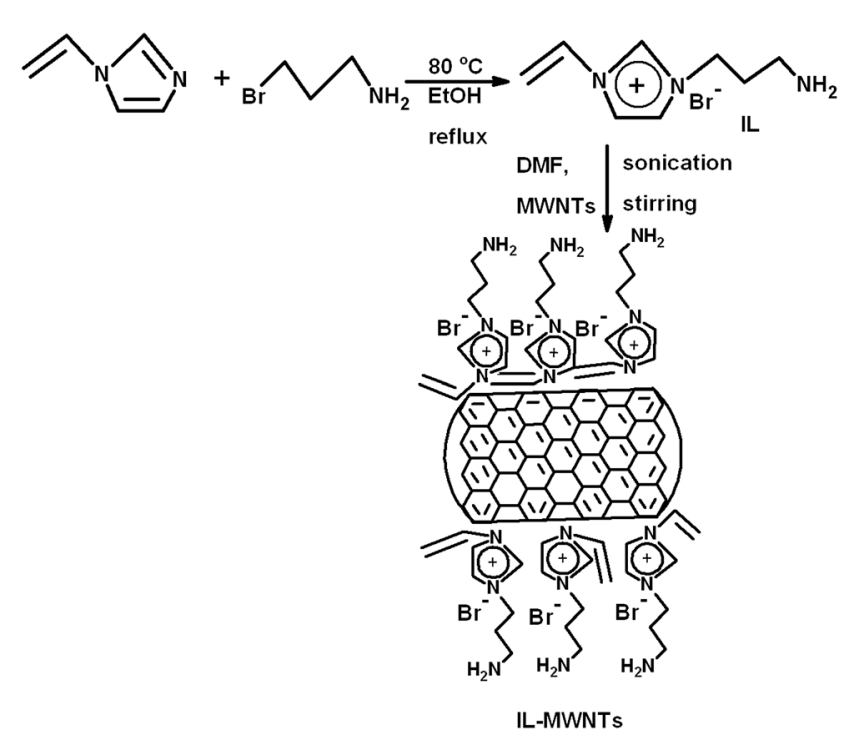

Scheme 1 Synthesis of $\mathrm{NH}_{2}$ terminated IL and IL-MWNTs. 
specimens were compression molded and the $S$ parameters $\left(S_{11}\right.$, $S_{12}, S_{22}$ and $S_{21}$ ) were measured in a wide range of frequencies.

\section{Results and discussion}

\section{Synthesis and characterization of IL-MWNTs and BT-GO}

Fig. 1(a) shows the FT-IR spectra of synthesized IL modified MWNTs. As shown in Scheme 1, amine terminated IL was synthesized by elimination reaction by refluxing under a nitrogen atmosphere for $24 \mathrm{~h}$. The direct evidence of the formation of amine terminated IL and the presence of IL on the surface of MWNTs is provided by FT-IR. The broad peak at 3385 $\mathrm{cm}^{-1}$ corresponding to the $\mathrm{N}-\mathrm{H}$ stretching clearly indicates the presence of the amine group attached to the IL. The peak at $3127 \mathrm{~cm}^{-1}$ corresponding to the $-\mathrm{C}=\mathrm{C}-\mathrm{H}$ stretching frequency supports the presence of the terminal alkene group. The peak at $2994 \mathrm{~cm}^{-1}$ indicates the $\mathrm{C}-\mathrm{H}$ stretching of alkanes. The peak at $1619 \mathrm{~cm}^{-1}$ indicates the $\mathrm{N}-\mathrm{H}$ bending, whereas the peak at 1460 $\mathrm{cm}^{-1}$ indicates the $\mathrm{C}-\mathrm{H}$ bending frequencies. The peak at 1246 $\mathrm{cm}^{-1}$ indicates the $\mathrm{C}-\mathrm{N}$ stretching frequency of the aliphatic amines. Further evidence of the grafting comes from the TEM micrograph and is discussed later on. The hydrophilic BT nanoparticles were initially refluxed with $\mathrm{H}_{2} \mathrm{O}_{2}$ to introduce the $\mathrm{O}-\mathrm{H}$ functional groups. The amino-silane coupling agent was then employed to terminate the BT nanoparticles with $\mathrm{NH}_{2}$
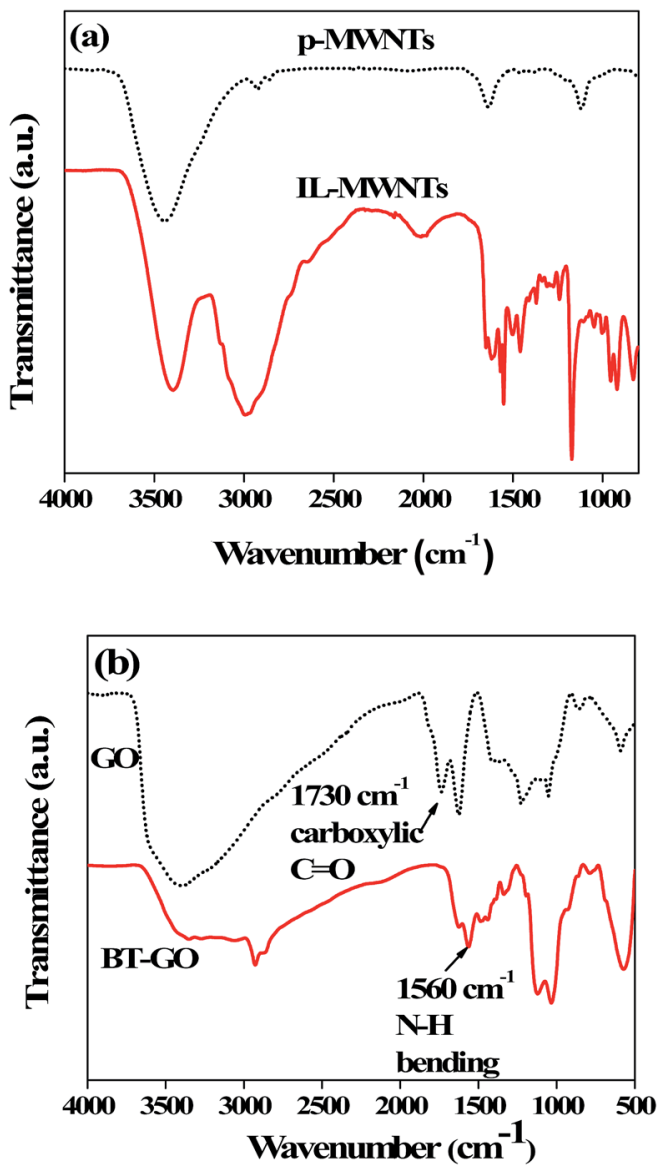

Fig. 1 FT-IR spectra of (a) p-MWNTs and IL modified MWNTs, and (b) $\mathrm{GO}$ and $\mathrm{BT}-\mathrm{GO}$. functional groups. The $\mathrm{NH}_{2}$ groups can readily react with the carboxyl $(\mathrm{C}=\mathrm{O})$ groups present on the basal planes of $\mathrm{GO}$ (see Scheme 2). The nature of interactions was assessed using FTIR. Fig. 1(b) shows the FTIR spectra of GO and BT-GO nanoparticles. The characteristic peaks of GO such as corresponding to hydroxyl (OH) stretching at $3400 \mathrm{~cm}^{-1}$, carboxylic stretching at $1730 \mathrm{~cm}^{-1}, \mathrm{C}=\mathrm{C}$ stretching at $1625 \mathrm{~cm}^{-1}, \mathrm{C}-\mathrm{OH}$ at 1220 $\mathrm{cm}^{-1}$ and $\mathrm{C}-\mathrm{O}$ stretching at $1055 \mathrm{~cm}^{-1}$ are well evident from the spectra. Interestingly, the carboxylic stretching at $1730 \mathrm{~cm}^{-1}$ is completely absent in $\mathrm{BT}-\mathrm{GO}$ indicating the amidation reaction between $\mathrm{C}=\mathrm{O}$ groups in $\mathrm{GO}$ and $\mathrm{BT}-\mathrm{NH}_{2}$. In addition, the appearance of a new band at $1560 \mathrm{~cm}^{-1}$ corresponding to the $\mathrm{N}-\mathrm{H}$ bending further confirms the reaction between $\mathrm{GO}$ and BT- $\mathrm{NH}_{2}$.

The TEM micrographs of IL modified MWNTs and BT-GO are shown in Fig. 2. The presence of IL as indicated in Fig. 2(a) is well discerned. Such observations were also reported earlier. ${ }^{36,37}$ Fig. 2(b) and (c) show the TEM micrographs of GO and BT-GO nanoparticles, respectively. It is well evident from Fig. 2(c) that the BT nanoparticles are stitched with the wrinkled GO nanosheets. Fig. 3(a) shows the TEM image of $\mathrm{Fe}_{3} \mathrm{O}_{4}$ nanoparticles, and Fig. 3(b) presents the magnetization hysteresis of $\mathrm{Fe}_{3} \mathrm{O}_{4}$ nanoparticles. From the hysteresis loop, a saturation magnetization $\left(M_{\mathrm{S}}\right)$ of $56 \mathrm{emu} \mathrm{g}^{-1}$ and remnant magnetization $\left(M_{\mathrm{R}}\right)$ of $22.5 \mathrm{emu} \mathrm{g}^{-1}$ are obtained. The coercive force $\left(H_{\mathrm{C}}\right)$ is observed to be 230 Oe.

\section{Selective localization of nanoparticles in the blends and the} phase morphology

Fig. 4 shows the SEM morphologies of the neat 50/50 (w/w) blends of PVDF/ABS and with different MWNTs. It is important to note that in all cases cryofractured blend samples have been etched with chloroform for $72 \mathrm{~h}$ at room temperature, which selectively removes the ABS phase from the blends. It is observed that the neat blends show a co-continuous type of morphology. Upon addition of $1 \mathrm{wt} \%$ p-MWNTs there is no significant change in the morphology, however, after the removal of the ABS phase the MWNTs are well observed in the remaining PVDF phase. This observation is in close harmony with the thermodynamic predictions and will be discussed later on. In addition, it is observed that the dispersion of MWNTs in the PVDF phase is significantly changed by the addition of $\mathrm{IL}$

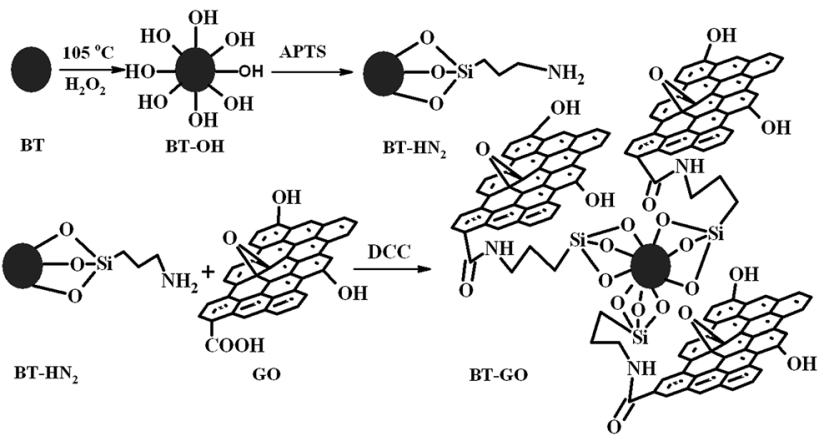

Scheme 2 Synthesis of BT-GO. 
modified MWNTs. In the case of p-MWNTs small clusters are observed in the PVDF phase (Fig. 4(c)) but on addition of IL modified MWNTs, relatively well dispersed MWNTs are noted (Fig. 4(e)). This observation clearly manifests the role of ionic liquids in facilitating uniform dispersion of MWNTs in the blends. Moreover, the dispersion state of IL modified MWNTs is also well supported by visual observation. For instance, the vial containing a known quantity of p-MWNTs settles quickly after brief sonication, whereas the IL modified MWNTs suspend quite well even for weeks (Fig. 2(a) inset vial picture). In order to further support the selective localization of MWNTs in the blends, solution dissolution experiments were performed. Samples of known amounts were dissolved in respective solvents to completely remove the ABS phase (chloroform) and the PVDF phase (DMF). It is important to note that though DMF is a common solvent to both PVDF and ABS, however, the kinetics of dissolution is quite different. PVDF readily dissolves in DMF, whereas ABS takes a few hours to dissolve. It is quite evident that vial 1 (chloroform) in the inset of Fig. 4(c) is colorless, whereas vial 2 (DMF) shows a strong black solution indicating that MWNTs preferentially localize in the PVDF phase of the blend. As a general observation, the blends with BT-GO and $\mathrm{Fe}_{3} \mathrm{O}_{4}$ showed similar co-continuous structures as seen in neat PVDF/ABS blends (Fig. 4(f) and (g)).

Selective localization of nanoparticles in a given phase or at the interface of a polymer blend can be predicted, a priori, from classical thermodynamics. This is accomplished by knowing the surface free energy values at the melt mixing temperature. By estimating the wetting coefficient (eqn (1)), shown here for the PVDF/ABS/MWNT system as an example, one can predict the localization of particles a priori. ${ }^{15}$

$$
\omega_{12}=\frac{\gamma_{\mathrm{T} 2}-\gamma_{\mathrm{T} 1}}{\gamma_{12}}
$$

where $\gamma_{\mathrm{T} 1}, \gamma_{\mathrm{T} 2}$, and $\gamma_{12}$ are the interfacial energies between MWNT/PVDF, MWNT/ABS and PVDF/ABS, respectively. If $\omega_{12}>$ 1 , MWNTs would selectively localize in the ABS phase, for $\omega_{12}<$ -1 , MWNTs would preferentially localize in the PVDF phase, and for $-1<\omega_{12}<1$, MWNTs would localize at the PVDF/ABS interface. The interfacial tension between the entities can be evaluated using the Owens-Wendt-Rabel-Kaelble (OWRK) equation and Wu's equation. The OWRK equation is often employed for nonpolar/polar systems and Wu's equation for nonpolar/nonpolar systems. ${ }^{38-40}$ In this study, the OWRK equation (eqn (2)) has been used to calculate the interfacial tension between PVDF and ABS.

$$
\gamma_{12}=\gamma_{1}+\gamma_{2}-2 \sqrt{\gamma_{1}^{\mathrm{d}} \gamma_{2}^{\mathrm{d}}}-2 \sqrt{\gamma_{1}^{\mathrm{p}} \gamma_{2}^{\mathrm{p}}}
$$

where $\gamma_{1}$ and $\gamma_{2}$ are the surface free energies of PVDF and ABS, respectively. $\gamma_{1}^{\mathrm{d}}$ and $\gamma_{2}^{\mathrm{d}}$ are disperse and $\gamma_{1}^{\mathrm{p}}$ and $\gamma_{2}^{\mathrm{p}}$ are the polar components of surface free energies of PVDF and ABS, respectively. The calculated $\gamma_{12}$ value of PVDF/ABS is $2.9 \mathrm{mN} \mathrm{m}^{-1}$. The surface free energy of MWNTs is $45.3 \mathrm{mN} \mathrm{m}^{-1}$ and $\omega_{12}$ is estimated to be -2.81 , which suggest that MWNTs prefer to localize in the PVDF phase of the blend. As a general understanding, nanoparticles which typically have high surface free energy tend to localize in the phase having higher surface free energy in order to minimize the interfacial tension. From the literature and the above calculations, it is well evident that PVDF has higher surface free energy as compared to ABS thereby we expect the other nanoparticles to localize in the PVDF phase similarly to MWNTs. ${ }^{41}$
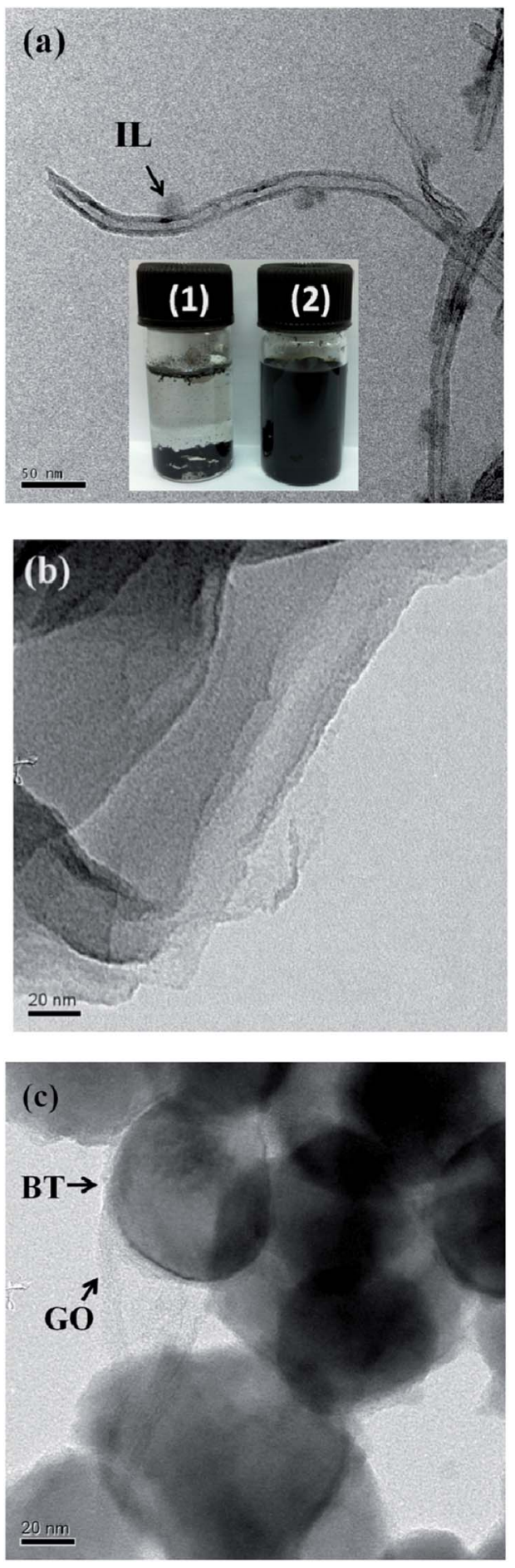

Fig. 2 TEM micrographs of IL-MWNTs (a), GO (b) and BT-GO (c). Inset of (a) shows the quality of dispersion of p-MWNTs (vial 1) and IL modified MWNTs (vial 2). 
Network like structures of MWNTs in the blends facilitated by IL: effect on electrical conductivity

The bulk electrical conductivity in the blends relies solely on the continuous conducting network of the conducting particles. Fig. 5(a) shows the AC electrical conductivity as a function of frequency for PVDF/ABS blends with MWNTs at room temperature. The percolation threshold for rod-like nanoparticles can be predicted by percolation theory $\left(P_{\mathrm{C}}=D / 2 L ; D\right.$ and $L$ correspond to the diameter and length of the filler), which clearly indicates that the percolation threshold $\left(P_{\mathrm{C}}\right)$ decreases with increasing aspect ratio of the conducting particles. For both the neat blends and with $0.5 \mathrm{wt} \%$ of p-MWNTs, the conductivity increases rapidly with increasing frequency manifesting a typical behavior of an insulator. Interestingly, the addition of 1 wt $\%$ p-MWNTs resulted in a six order increment in the AC conductivity, with respect to the neat as well as blends with 0.5 wt\% MWNTs. This phenomenal increase in conductivity can be attributed to the gelation of p-MWNTs in the PVDF phase beyond $0.5 \mathrm{wt} \%$ MWNTs in the blends. Further, it was observed that the AC electrical conductivity scales with the concentration of the MWNT frequency independent plateau extended to higher frequency. Interestingly, in the presence of $1 \mathrm{wt} \% \mathrm{IL}$ modified MWNTs the AC electrical conductivity increased further with respect to $1 \mathrm{wt} \% \mathrm{p}$-MWNTs and more interestingly represented a similar electrical conductivity to that of $3 \mathrm{wt} \% \mathrm{p}$ MWNTs (Fig. 5(a)). The dramatic increase in the AC electrical conductivity is due to the network-like structure of MWNTs in the PVDF phase facilitated by IL. Blends with $\mathrm{Fe}_{3} \mathrm{O}_{4}$ and BT-GO nanoparticles showed more or less similar type of AC conductivity with respect to blend with 1 wt\% IL-MWNTs clearly suggesting that the network like structure of MWNTs is largely unaffected by the presence of BT-GO and $\mathrm{Fe}_{3} \mathrm{O}_{4}$ (Fig. 5(b)). Fig. 5(c) shows the frequency dependent dielectric constant $\left(\varepsilon^{\prime}\right)$ of the various blends. Dielectric properties stem from the uneven polarization of electrons in an external electric field. Complex permittivity is given as, ${ }^{42} \varepsilon^{*}=\varepsilon^{\prime}-\mathrm{i} \varepsilon^{\prime \prime}$, where $\varepsilon^{\prime}$ and $\varepsilon^{\prime \prime}$ are the real and imaginary part of the complex permittivity. $\varepsilon^{\prime}$ and $\varepsilon^{\prime \prime}$ depend on the frequency, $\omega$ in the applied electric field and can be expressed as, ${ }^{\mathbf{4 3}}$

$$
\varepsilon^{\prime}(\omega)=\varepsilon_{0}+\frac{2}{\pi} \int_{0}^{\infty} \frac{u \varepsilon^{\prime \prime}(u)}{u^{2}-\omega^{2}} \mathrm{~d} u
$$

Due to blending a low $k$ material (ABS) with a high $k$ material (PVDF), the dielectric constant decreases with respect to the neat PVDF. ${ }^{44}$ At $100 \mathrm{~Hz}$ the 50/50 PVDF/ABS neat blends show an $\varepsilon^{\prime}$ of 5 . In the presence of $0.1 \% \mathrm{GO}$, the $\varepsilon^{\prime}$ increases to 6.5 . However, in the presence of BT nanoparticles, it further increases to 9.7. Interestingly, functionalization of BT with GO enhances the polarization further. This is manifested by a significant increase in $\varepsilon^{\prime}$ to 12 in the blends with only 2 vol\% BT-GO particles.

\section{Attenuation of EM radiation: effect of $\mathrm{BT}-\mathrm{GO}$ and $\mathrm{Fe}_{3} \mathrm{O}_{4}$}

The spectrum of electromagnetic radiation is composed of waves with electric and magnetic components oscillating at right angles to each other. The electromagnetic shielding is a process of limiting the flow of electromagnetic fields between two locations, by separating them with a barrier made of conducting materials. So EMI shielding effectiveness (SE) can be enhanced by different ways in polymer composites. Sometimes attenuation of EM radiation can be done by either addition of conducting nanoparticles to an insulating polymer matrix or with an inherently conducting polymer as the matrix. Materials with high magnetic permeability are also used to attenuate the incident $\mathrm{EM}$ radiation. The shielding effectiveness is the amount of attenuation of incident radiation by a particular material and is expressed in $\mathrm{dB}$. There are three mechanisms in shielding, namely reflection $\left(\mathrm{SE}_{\mathrm{R}}\right)$, absorption $\left(\mathrm{SE}_{\mathrm{A}}\right)$ and multiple reflections $\left(\mathrm{SE}_{\mathrm{MR}}\right)$. The multiple reflections can be ignored when $\mathrm{SE}_{\mathrm{T}}$ is greater than $15 \mathrm{~dB}^{45}$ The reflection mechanism is prominent in the case of materials containing free mobile charge carriers. In order to reflect EM radiation, materials should have high electrical conductivity and mobile charge carriers which can interact with the incident EM radiation. EM radiation attenuated by absorption requires both electric and magnetic dipoles. This absorption loss arises due to generation of eddy currents which is the result of ohmic losses and leads to heating of the material. Owing to the high thermal
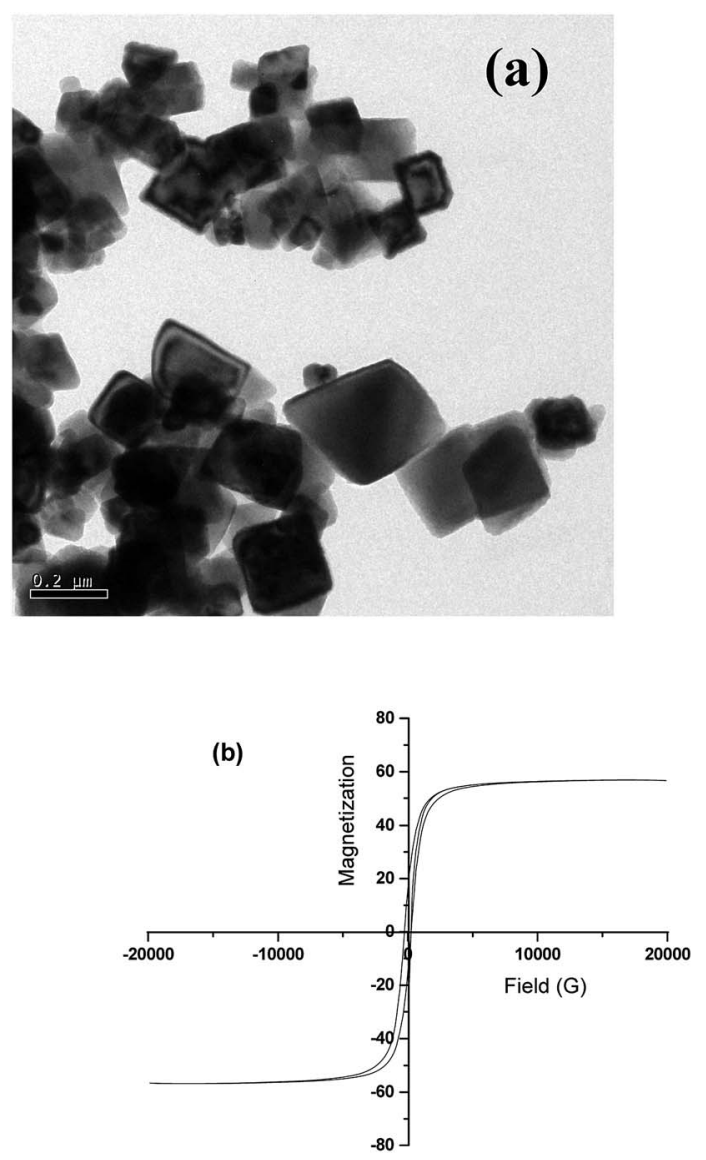

Fig. 3 TEM micrographs of $\mathrm{Fe}_{3} \mathrm{O}_{4}$ nanoparticles (a) and the magnetic hysteresis loop with applied magnetic field at room temperature of $\mathrm{Fe}_{3} \mathrm{O}_{4}$ nanoparticles (b). 

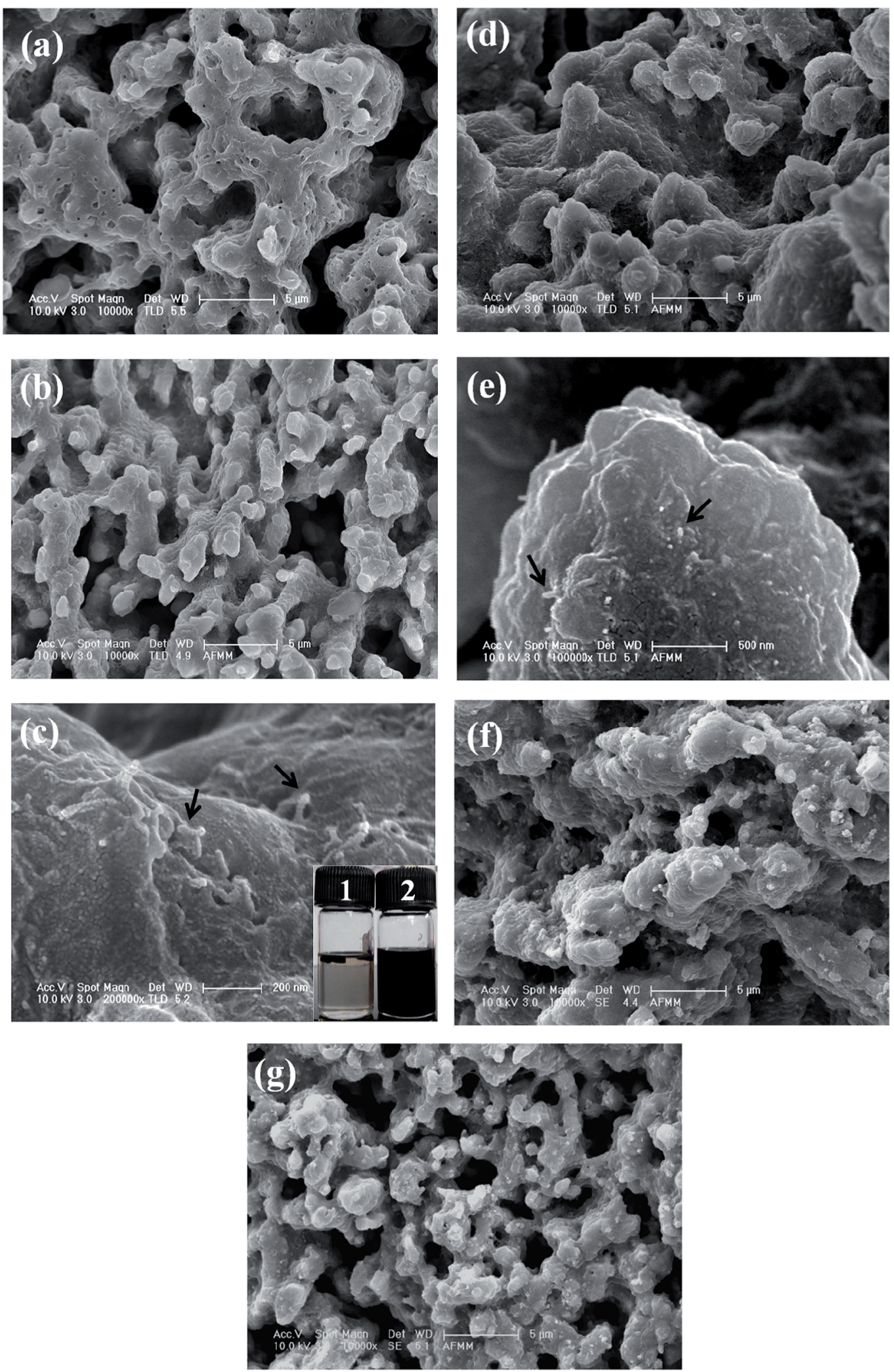

Fig. 4 SEM morphologies of 50/50 PVDF/ABS neat blends (a), with 1\% p-MWNTs (b), high magnification with $1 \%$ p-MWNTs (c) (inset vial picture in $\mathrm{CHCl}_{3}$ and in DMF), with $1 \%$ IL-MWNTs (d), high magnification with $1 \%$ IL-MWNTs (e), with IL-MWNTs and Fe ${ }_{3} \mathrm{O}_{4}$ (f) and IL-MWNTs with $\mathrm{BT}-\mathrm{GO}(\mathrm{g})$. 
conductivity of MWNTs, heat generated due to ohmic losses can be dissipated easily through a MWNT network.

The total SE is the summation of shielding by absorption $\left(\mathrm{SE}_{\mathrm{A}}\right)$, reflection $\left(\mathrm{SE}_{\mathrm{R}}\right)$ and multiple reflections $\left(\mathrm{SE}_{\mathrm{MR}}\right)$ and is expressed as $\mathrm{SE}_{\mathrm{T}}=\mathrm{SE}_{\mathrm{A}}+\mathrm{SE}_{\mathrm{R}}+\mathrm{SE}_{\mathrm{MR}}$ and is the ratio of $\mathrm{EM}$ radiation transmitted through the sample to incident $\mathrm{EM}$ radiation

$$
\operatorname{SET}(\mathrm{dB})=-20 \log \frac{E_{\mathrm{T}}}{E_{\mathrm{I}}}=-20 \log \frac{H_{\mathrm{T}}}{H_{\mathrm{I}}}=-20 \log \frac{P_{\mathrm{T}}}{P_{\mathrm{I}}}
$$

where, $E, H$ and $P$ are transmitted electric, magnetic and electromagnetic power, respectively. Subscripts $\mathrm{T}$ and I correspond to transmission and incident parts of the radiation. In a vector network analyser, the EMI SE is represented in terms of scattering parameters which are $S_{11}, S_{12}$, and $S_{21}$. The total EMI SE can be evaluated from the $S$ parameter using the following equation, ${ }^{46-48}$

$$
\operatorname{SET}(\mathrm{dB})=10 \log \frac{1}{\left|S_{12}\right|^{2}}=10 \log \frac{1}{\left|S_{21}\right|^{2}}
$$

SE due to different mechanisms, reflection and absorption mentioned above can be evaluated by the following equations,

$$
\begin{gathered}
\mathrm{SE}_{\mathrm{R}}=10 \log _{10}\left(1 /\left(1-S_{11}^{2}\right)\right) \\
\mathrm{SE}_{\mathrm{A}}=10 \log _{10}\left(\left(1-{S_{11}}^{2}\right) / S_{12}{ }^{2}\right)
\end{gathered}
$$

where scattering parameters $S_{11}, S_{12}$ and $S_{21}$ are termed as forward reflection coefficient, reverse transmission coefficient and forward transmission coefficient, respectively.

As we discussed earlier, the conductivity and network formation of MWNTs are the key requirements to achieve high attenuation of EM radiation. It is well established that by selectively localizing MWNTs in a given phase of the blend, network like structures of MWNTs can be obtained. All the experiments were performed in the $\mathrm{X}$ and $\mathrm{Ku}$-band frequency range. These frequency ranges are quite important for commercial applications like TV signal transmission, Doppler, telephone radiation, radar systems, etc.

Fig. 6(a) shows the EMI shielding performances of the different blends. It was observed that PVDF/ABS blends with1 wt\% pMWNTs show a SE of $-16 \mathrm{~dB}$ while the neat blends and with 0.5 wt\% p-MWNTs are transparent to EM radiation. It is well evident that the blends with insulating characteristics are transparent to incident radiation but conducting networks formed due to the addition of MWNTs beyond the percolation threshold interact with incident rays and account for shielding effectiveness. The EMI SE increased from $-16 \mathrm{~dB}$ to $-23 \mathrm{~dB}$ at $18 \mathrm{GHz}$ with increasing concentration of p-MWNTs from 1 to $3 \mathrm{wt} \%$. Interestingly, with the addition of $1 \mathrm{wt} \%$ IL-MWNTs the SE is $-22 \mathrm{~dB}$ at 18 GHz which clearly manifests the key role of ionic liquid in facilitating the formation of an interconnected network structure of MWNTs and attenuating more EM radiation. As we discussed earlier reflection, absorption and multiple reflection are the mechanisms of shielding, where reflection is the primary mechanism and absorption is the secondary mechanism in the case of uniformly dispersed conducting nanoparticles. After the addition of $2 \mathrm{vol} \% \mathrm{Fe}_{3} \mathrm{O}_{4}$ along with $1 \mathrm{wt} \%$ IL-MWNTs, there is no significant improvement in the SE whereas we observed $c a .4 \mathrm{~dB}$ increment after the addition of 2 vol\% BT-GO particles. Moreover,
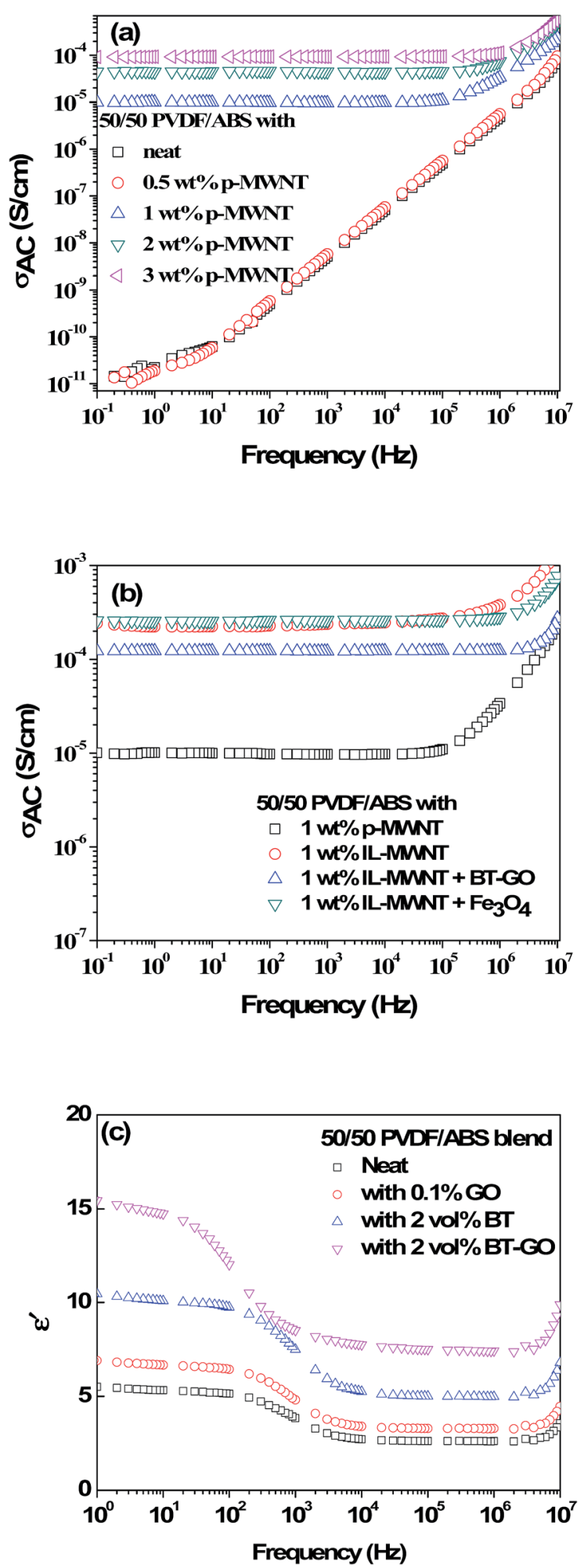

Fig. 5 AC electrical conductivity of blends (a) with different wt\% of MWNTs and (b) with IL-MWNTs along with inorganic nanoparticles. (c) Frequency dependent dielectric constant of various blends. 

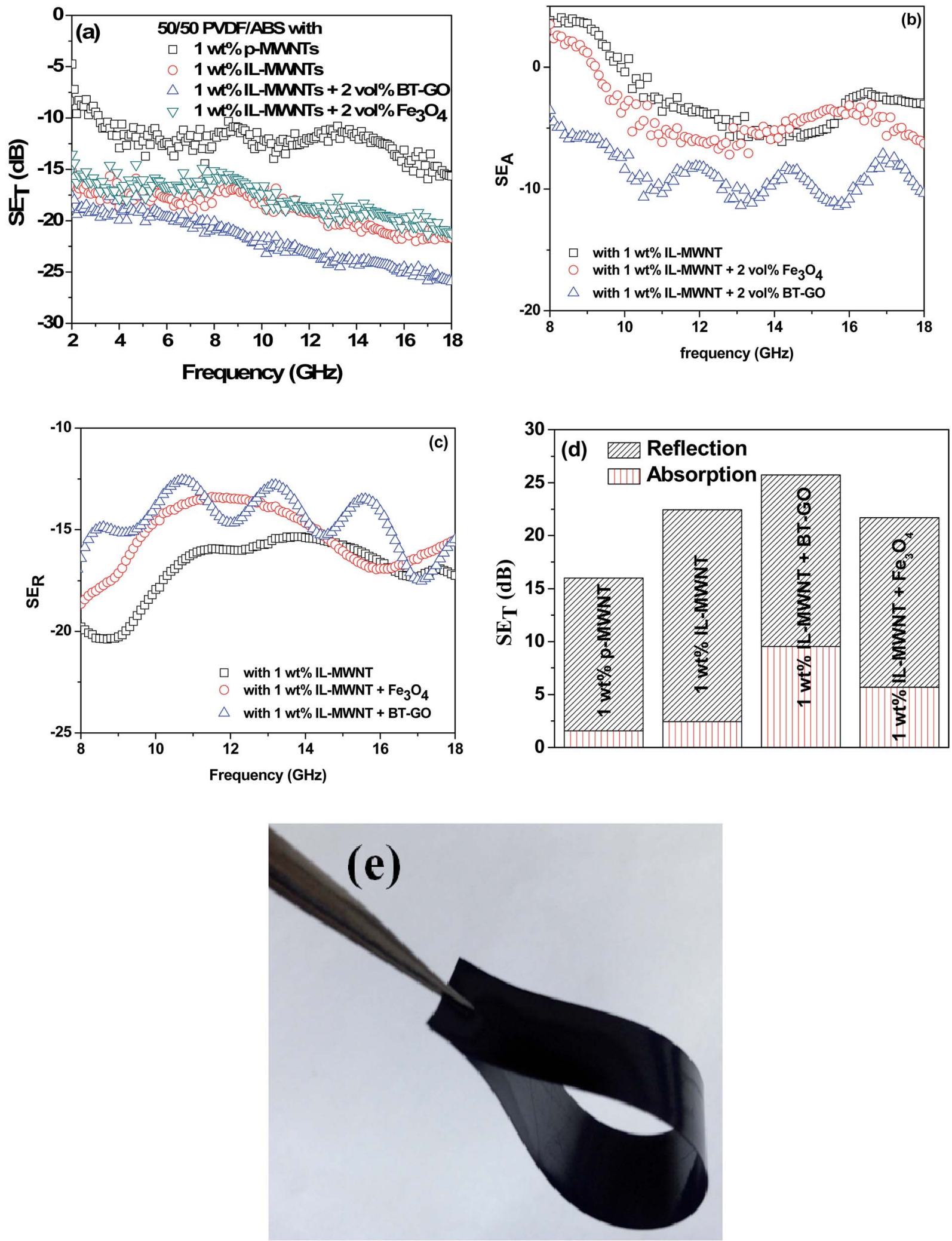

Fig. 6 (a-c) EMI shielding effectiveness (SE) of the various blends, (d) reflection and absorption components in the blends, and (e) twisted film of the blend with 1 wt\% IL modified MWNTs and BT-GO showing the flexibility of the film.

absorption is the governing mechanism of shielding in the presence of BT-GO nanoparticles (Fig. 6(b) and (c)). As discussed earlier, the high dielectric constant materials like BT-GO can attenuate EM radiation. It is worth mentioning here that PVDF/ BT-GO exhibited a significantly higher dielectric constant than both PVDF/GO and PVDF/BT composites which is illustrated in 
Table 1 EM shielding parameters for 50/50 PVDF/ABS blends with different nanoparticles at $18 \mathrm{GHz}$

\begin{tabular}{llll}
\hline Compositions & $\mathrm{SE}_{\mathrm{T}}(\mathrm{dB})$ & $\mathrm{SE}_{\mathrm{A}}(\mathrm{dB})$ & $\mathrm{SE}_{\mathrm{R}}(\mathrm{dB})$ \\
\hline With 1 wt\% p-MWNT & 16 & 1.5 & 14.5 \\
With 2 wt\% p-MWNT & 19 & 2 & 17 \\
With 3 wt\% p-MWNT & 23 & 2.8 & 20.2 \\
With 1 wt\% IL-MWNT & 22 & 2.9 & 19.1 \\
With 1 wt\% IL-MWNT + 2 vol\% Fe $\mathrm{O}_{4}$ & 22 & 6.8 & 15.2 \\
With 1 wt\% IL-MWNT + 2 vol\% GO & 22 & 5 & 17 \\
With 1 wt\% IL-MWNT + 2 vol\% BT-NH & 23 & 6 & 17 \\
With 1 wt\% IL-MWNT + 2 vol\% BT-GO & 26 & 10.5 & 15.5
\end{tabular}

Fig. 5(c). ${ }^{49}$ A comparison has been made to highlight the effects of BT-GO towards attenuating EM radiation (Fig. 6(d) and Table 1). A twisted film of the blend with 1 wt\% IL modified MWNTs and BTGO is depicted in Fig. 6(e). This clearly shows that the flexibility of the film is retained even at 2 vol\% BT-GO nanoparticles.
In order to get more insight into the mechanism of shielding, we took a closer look at the permeability and permittivity values. Well-established line theory proposed by Nicolson and Ross was adopted to estimate the permeability and permittivity of the blends. It is observed that the permeability in the case of $\mathrm{Fe}_{3} \mathrm{O}_{4}$ containing blend is higher, and the permittivity is maximum in BT-GO containing blends. Hence, the enhanced shielding effectiveness is mainly due to the improved permittivity and permeability values in the blends (Fig. 7(a) and (b)).

We also calculated the reflection loss by line theory proposed by Nicolson and Ross. We observed that the blends containing BT-GO showed a minimum RL $(-44.9 \mathrm{~dB})$ at $15.9 \mathrm{GHz}$ and the blend containing $\mathrm{Fe}_{3} \mathrm{O}_{4}$ showed a minimum $\mathrm{RL}(-38.7 \mathrm{~dB})$ at 17 GHz (Fig. 7(c)).

It is well accepted in the literature that EM shielding efficiency is dependent on the sample thickness. We varied the thickness of the samples and observed that the shielding effectiveness increases significantly (Fig. 7(d)) beyond certain
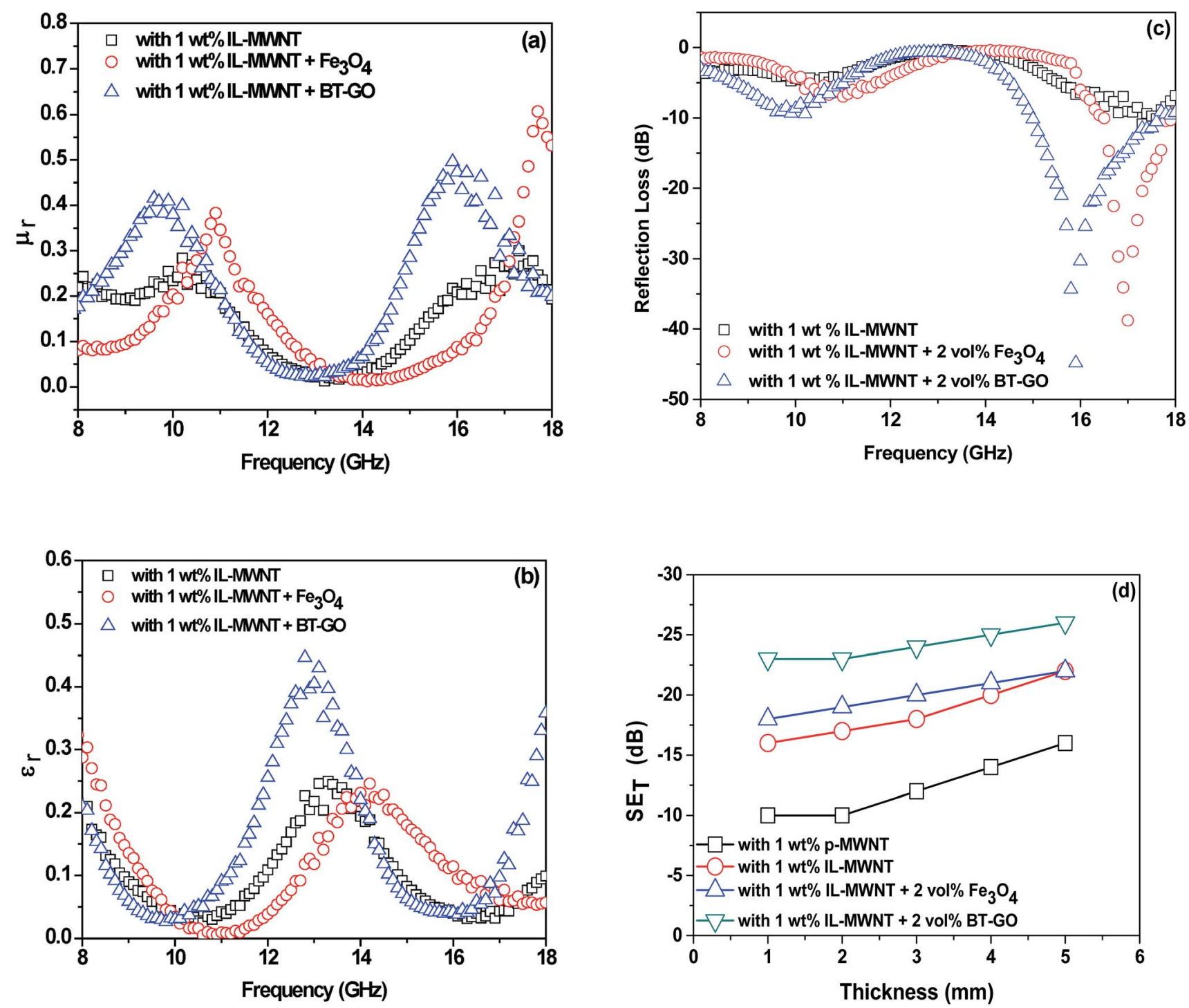

Fig. 7 (a) Permeability; (b) permittivity; (c) reflection loss; (d) shielding effectiveness as a function of shield thickness in PVDF/ABS blends. 
Table 2 EM shielding parameters for various composites at the same thickness (5 $\mathrm{mm})$

\begin{tabular}{|c|c|c|c|c|c|}
\hline System & Conducting fillers & Concentration & Frequency (GHz) & $\mathrm{SE}_{\mathrm{T}}(\mathrm{dB})$ & Reference \\
\hline $\mathrm{PC} / \mathrm{ABS}$ & MWNTs & $3 \mathrm{phr}$ & 1.5 & -7 & 51 \\
\hline PS/PMMA & Amine terminated MWNT & $1 \mathrm{wt} \%$ & 18 & -26 & 15 \\
\hline PC/SAN & MWNT- $g-\mathrm{Fe}_{3} \mathrm{O}_{4}$ & $3 w t \%$ & 18 & -32 & 18 \\
\hline PVDF & $f$-Graphene & $7 \mathrm{wt} \%$ & 12 & -18.5 & 53 \\
\hline PVDF & IL-MWNTs & $2 \mathrm{wt} \%$ & 18 & -20 & 26 \\
\hline PVDF/ABS & IL-MWNT + BT-GO & $1 \mathrm{wt} \%$ & 18 & -26 & This work \\
\hline
\end{tabular}

thickness $(2 \mathrm{~mm})$. Generally, the formation of a conductive mesh in the matrix intercepts the electromagnetic radiation. Now with increase in the shield thickness, the number of conductive meshes increases resulting in enhanced EMI SE. ${ }^{\mathbf{5 0 , 5 2}}$

The SE obtained in this study is quite high especially given the concentration of the MWNTs being quite low. It is clearly evident from the literature (see Table 2) that higher loading of conducting fillers can lead to higher attenuation of EM radiation. But it is also important to note that higher loading of conducting fillers can lead to adverse effects on the structural properties of the nanocomposites and is also difficult to process. In this study, we have successfully improved the SE at a very low fraction of MWNTs via modification of ionic liquid. It is well evident that dispersion of MWNTs is the key factor to improve the EM attenuation besides the presence of ferroelectric (BT-GO) and ferromagnetic phases $\left(\mathrm{Fe}_{3} \mathrm{O}_{4}\right)$.

\section{Conclusions}

In this work, different MWNTs were incorporated into PVDF/ ABS blends to design materials with high EMI SE. The selective localization and dispersion of p-MWNTs were successfully tuned by the addition of ionic liquid which also helped in the improvement in bulk electrical conductivity and EMI SE. The EMI shielding in $\mathrm{X}$ and $\mathrm{Ku}$ band frequency was assessed here and the blends with 1 wt $\%$ IL modified MWNTs showed significant improvement in SE. The EM attenuation through absorption was enhanced by incorporation of BT-GO nanoparticles along with $1 \mathrm{wt} \%$ IL modified MWNTs and interestingly attained $26 \mathrm{~dB}$ at $18 \mathrm{GHz}$ frequency. Such results can be quite interesting in designing lightweight and flexible materials for microwave absorption with addition of a very lower concentration of nanofillers.

\section{Acknowledgements}

The authors gratefully acknowledge the financial support from DST (India).

\section{References}

1 L. A. Utracki, Polymer blends handbook, Kluwer Academic Publishers, Dordrecht, The Netherlands, 2002.
2 G. P. Kar, N. Begam, J. K. Basu and S. Bose, Macromolecules, 2014, 47, 7525-7532.

3 G. P. Kar, P. Xavier and S. Bose, Phys. Chem. Chem. Phys, 2014, 16, 17811-17821.

4 A. Bharati, P. Xavier, G. P. Kar, G. Madras and S. Bose, J. Phys. Chem. B, 2014, 118, 2214-2225.

5 G. P. Kar, A. Bharati, P. Xavier, G. Madras and S. Bose, Phys. Chem. Chem. Phys., 2015, 17, 868-877.

6 S. Bose, A. R. Bhattacharyya, A. R. Kulkarni and P. Pötschke, Compos. Sci. Technol., 2009, 69, 365-372.

7 A. M. Stephan, Eur. Polym. J., 2006, 42, 21-42.

8 G. P. Kar, S. Biswas and S. Bose, Phys. Chem. Chem. Phys., 2015, 17, 1811-1821.

9 S. Bose, A. R. Bhattacharyya, R. A. Khare, S. S. Kamath and A. R. Kulkarni, Polym. Eng. Sci., 2011, 51, 1987-2000.

10 S. Bose, R. A. Khare and P. Moldenaers, Polymer, 2010, 51, 975-993.

11 P. V. Kodgire, A. R. Bhattacharyya, S. Bose, N. Gupta, A. R. Kulkarni and A. Misra, Chem. Phys. Lett., 2006, 432, 480-485.

12 S. Bose, A. R. Bhattacharyya, M. Chawley, P. V. Kodgire, A. R. Kulkarni, A. Misra and P. Poetschke, J. Nanosci. Nanotechnol., 2008, 8, 1721-1727.

13 S. Bose, A. R. Bhattacharyya, R. A. Khare, A. R. Kulkarni and P. Poetschke, Macromol. Symp., 2008, 263, 11-20.

14 S. Bose, A. R. Bhattacharyya, R. A. Khare, S. S. Kamath and A. R. Kulkarni, Polym. Eng. Sci., 2011, 51, 1987-2000.

15 R. Rohini and S. Bose, ACS Appl. Mater. Interfaces, 2014, 6, 11302-11310.

16 P. Xavier and S. Bose, Rsc Adv., 2014, 4, 55341-55348.

17 S. P. Pawar, K. Pattabhi and S. Bose, RSC Adv., 2014, 4, 18842-18852.

18 S. P. Pawar, D. Marathe, K. Pattabhi and S. Bose, J. Mater. Chem. A, 2015, 3, 656-669.

19 D. D. L. Chung, Carbon, 2001, 39, 279-285.

20 N. Li, Y. Huang, F. Du, X. B. He, X. Lin, H. J. Gao, Y. F. Ma, F. F. Li, Y. S. Chen and P. C. Eklund, Nano Lett., 2006, 6, 1141-1145.

21 Y. Y. Wang and X. L. Jing, Polym. Adv. Technol., 2005, 16, 344351.

22 S. Bhadra, D. Khastgir, N. K. Singha and J. H. Lee, Prog. Polym. Sci., 2009, 34, 783-810. 
23 B. P. Singh, Prabha, P. Saini, T. Gupta, P. Garg, G. Kumar, I. Pande, S. Pande, R. K. Seth, S. K. Dhawan and R. B. Mathur, J. Nanopart. Res., 2011, 13, 7065-7074.

24 M. H. Al-Saleh and U. Sundararaj, Carbon, 2009, 47, 17381746.

25 Z. Liu, G. Bai, Y. Huang, Y. Ma, F. Du, F. Li, T. Guo and Y. Chen, Carbon, 2007, 45, 821-827.

26 M. Sharma, S. Sharma, J. Abraham, S. Thomas, G. Madras and S. Bose, Mater. Res. Express, 2014, 1, 035003.

27 R. K. Donato, K. Z. Donato, H. S. Schrekker and L. Matejka,J. Mater. Chem., 2012, 22, 9939-9948.

28 R. K. Donato, L. Matejka, H. S. Schrekker, J. Plestil, A. Jigounov, J. Brus and M. Slouf, J. Mater. Chem., 2011, 21, 13801-13810.

29 J. Dupont, R. F. de Souza and P. A. Z. Suarez, Chem. Rev., 2002, 102, 3667-3691.

30 R. D. Rogers, Nature, 2007, 447, 917-918.

31 T. Fukushima and T. Aida, Chem.-Eur. J., 2007, 13, 50485058.

32 A. Mandal and A. K. Nandi, ACS Appl. Mater. Interfaces, 2013, 5, 747-760.

33 P. K. S. Mural, A. Banerjee, M. S. Rana, A. Shukla, B. Padmanabhan, S. Bhadra, G. Madras and S. Bose, J. Mater. Chem. A, 2014, 2, 17635-17648.

34 S. Bose, H. Raghu and P. A. Mahanwar, J. Appl. Polym. Sci., 2006, 100, 4074-4081.

35 L. Xie, X. Huang, C. Wu and P. Jiang, J. Mater. Chem., 2011, 21, 5897-5906.

36 B. Yu, F. Zhou, G. Liu, Y. Liang, W. T. S. Huck and W. Liu, Chem. Commun., 2006, 2356-2358.

37 B. Wu, D. Hu, Y. Yu, Y. Kuang, X. Zhang and J. Chen, Chem. Commun., 2010, 46, 7954-7956.
38 D. F. Coutinho, I. H. Pashkuleva, C. M. Alves, A. P. Marques, N. M. Neves and R. L. Reis, Biomacromolecules, 2008, 9, 11391145.

39 Q. Zhao, Y. Liu and E. W. Abel, J. Colloid Interface Sci., 2004, 280, 174-183.

40 X. Sun, Y. Liu, C.-a. Di, Y. Wen, Y. Guo, L. Zhang, Y. Zhao and G. Yu, Adv. Mater., 2011, 23, 1009-1014.

41 X. Zhao, J. Zhao, J.-P. Cao, X. Wang, M. Chen and Z.-M. Dang, J. Phys. Chem. B, 2013, 117, 2505-2515.

42 J.-K. Yuan, S.-H. Yao, A. Sylvestre and J. Bai, J. Phys. Chem. C, 2011, 116, 2051-2058.

43 P. Barber, S. Balasubramanian, Y. Anguchamy, S. Gong, A. Wibowo, H. Gao, H. J. Ploehn and H.-C. Zur Loye, Materials, 2009, 2, 1697-1733.

44 M. Sharma, G. Madras and S. Bose, Macromolecules, 2014, 47, 1392-1402.

45 N. Li, Y. Huang, F. Du, X. He, X. Lin, H. Gao, Y. Ma, F. Li, Y. Chen and P. C. Eklund, Nano Lett., 2006, 6, 1141-1145.

46 N. F. Colaneri and L. W. Shacklette, IEEE Trans. Electromagn. Compat., 1992, 41, 291-297.

47 S. K. Dhawan, N. Singh and S. Venkatachalam, Synth. Met., 2002, 129, 261-267.

48 R. B. Schulz, V. C. Plantz and D. R. Brush, IEEE Trans. Electromagn. Compat., 1988, 30, 187-201.

49 M. Sharma, M. P. Singh, C. Srivastava, G. Madras and S. Bose, ACS Appl. Mater. Interfaces, 2014, 6, 21151-21160.

50 M. Rahaman, T. K. Chaki and D. Khastgir, J. Mater. Sci., 2011, 46, 3989-3999.

51 I.-S. Han, Y. Lee, H. Lee, H. Yoon and W. Kim, J. Mater. Sci., 2014, 49, 4522-4529.

52 M. H. Al-Saleha, W. H. Saadeha and U. Sundararaj, Carbon, 2013, 60, 146-156.

53 V. Eswaraiah, V. Sankaranarayanan and S. Ramaprabhu, Macromol. Mater. Eng., 2011, 296, 894-898. 